\title{
Exploratory phase II trial in a multicenter setting to evaluate the clinical value of a chemosensitivity test in patients with gastric cancer (JACCRO-GC 04, Kubota memorial trial)
}

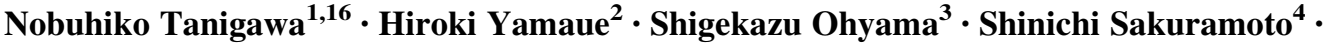 \\ Takao Inada $^{5} \cdot$ Yasuhiro Kodera $^{6} \cdot$ Yuko Kitagawa $^{7} \cdot$ Kenji Omura $^{8}$. \\ Masanori Terashima ${ }^{9} \cdot$ Yuh Sakata $^{10}$ - Atsushi Nashimoto ${ }^{11}$. Toshiharu Yamaguchi ${ }^{3}$. \\ Keisho Chin $^{12} \cdot$ Eiji Nomura $^{1} \cdot$ San-Woong Lee ${ }^{1} \cdot$ Masahiro Takeuchi $^{13}$. \\ Masashi Fujii ${ }^{14}$ - Toshifusa Nakajima ${ }^{15}$
}

Received: 10 March 2015/Accepted: 22 May 2015/Published online: 18 September 2015

(C) The Author(s) 2015. This article is published with open access at Springerlink.com

\begin{abstract}
Background Although postoperative adjuvant chemotherapy with S-1, an oral fluoropyrimidine, has become a standard of care for gastric cancer in Japan, nonresponders may suffer from the cost and adverse reactions without clinical benefit. This multicenter exploratory phase II trial was conducted to see whether a chemosensitivity test, the collagen gel droplet embedded culture drug sensitivity
\end{abstract}

For the JACCRO-GC04 Group.

The investigators in the Japan Clinical Cancer Research Organization Gastric Cancer 04 (JACCRO GC-04) Group are listed in the Appendix.

Presented in part at the European Society for Medical Oncology Congress 2014, Madrid, Spain, 26-30 September 2014.

Nobuhiko Tanigawa

sur001@poh.osaka-med.ac.jp; tanigawa@tanigawa-hp.or.jp

1 Department of General and Gastroenterological Surgery, Osaka Medical College, Osaka, Japan

2 Second Department of Surgery, Wakayama Medical University, Wakayama, Japan

3 Division of Digestive Surgery, Cancer Center Institute Ariake Hospital, Tokyo, Japan

4 Departmet of Surgery, Kitasato University, Sagamihara, Tokyo, Japan

5 Division of Surgery, Tochigi Cancer Center, Utsunomiya, Japan

6 Second Department of Surgery, Nagoya University Graduate School of Medicine, Nagoya, Japan

7 Department of Surgery, Faculty of Medicine, Keio University, Tokyo, Japan test (CD-DST), can adequately select patients for chemotherapy.

Methods The CD-DST using four different concentrations of 5-fluorouracil was conducted with resected specimens from preregistered patients who underwent gastrectomy with D2 or more extensive lymphadenectomy. Patients who were histopathologically confirmed to have stage II or greater disease without distant metastasis were eligible for final enrollment. All patients underwent protocol-specified adjuvant chemotherapy with S-1. Threeyear relapse-free survival was compared between patients determined as sensitive by the CD-DST (responders) and those deemed insensitive (nonresponders). Appropriate cutoff values for in vitro growth inhibition were defined when the hazard ratio for relapse in responders and the logrank $P$ values were at their minimum.

8 Division of Surgery, Ageo Central General Hospital, Ageo, Japan

9 Division of Surgery, Shizuoka Cancer Center, Shizuoka, Japan

10 Misawa City Hospital, Aomori, Japan

11 Division of Surgery, Niigata Cancer Center Hospital, Niigata, Japan

12 Division of Medical Oncology, Cancer Center Institute Ariake Hospital, Tokyo, Japan

13 Department of Pharmacology, Kitasato University, Sagamihara, Japan

14 Department of Surgery, Nihon University of School of Medicine, Tokyo, Japan

15 The Japan Clinical Cancer Research Organization, Tokyo, Japan

16 Tanigawa Memorial Hospital, 16-59 Kasuga 1-Cho-Me, Ibaraki, Osaka 567-0031, Japan 
Results Of the 311 patients enrolled, 14 were ineligible and 27 failed to start the protocol treatment. The CD-DST failed in 64 other patients, and survival analyses were conducted with the remaining 206 patients (39 stage II disease, 155 stage III disease, and 12 stage IV disease). The outcome of patients who were determined to be responders was significantly superior to that of nonresponders regardless of the 5-fluorouracil concentrations, although no differences in clinicopathologic characteristics were observed between the two groups, except for age.

Conclusions The CD-DST identified those who benefit from adjuvant chemotherapy. It deserves further evaluation in the setting of a prospective randomized trial.

ClinicalTrials.gov identifier: NCT00287755

Keywords Chemosensitivity test - Relapse-free survival . Appropriate cutoff values $\cdot$ Responder $\cdot$ Nonresponder

\section{Introduction}

The outcome of patients with resectable gastric cancer has improved owing to the development of technologies making possible earlier diagnosis, as well as the continued progress in surgical techniques and multidisciplinary treatments. However, the outcome remains unsatisfactory in patients with advanced or recurrent disease. Recently, several anticancer agents have been newly introduced, and have raised hope for an improved outcome after chemotherapy. S-1 (TS-1, Taiho Pharmaceutical, Tokyo, Japan) is an oral anticancer drug that combines tegafur (a prodrug of 5-fluorouracil; 5-FU) with 5-chloro-2,4-dihydropyrimidine (CDHP) and potassium oxonate in a molar ratio of 1:0.4:1. A phase III study comparing surgical treatment alone with surgery plus adjuvant S-1 chemotherapy in patients who underwent curative resection of stage II and stage III gastric cancer (Adjuvant Chemotherapy Trial of TS-1 for Gastric Cancer; ACTS-GC) demonstrated that postoperative adjuvant chemotherapy with S-1 significantly improved survival [1, 2]. However, human tumors of even a similar histopathologic category may have markedly different drug sensitivity profiles [36]. In vitro drug sensitivity tests have thus been developed to individualize chemotherapy for cancer patients [7-15]. We hypothesize that personalized therapy guided by adequate chemosensitivity testing may lead to a better outcome than conventional empirical therapy. Since the publication of ACTS-GC, orally administered S-1 has become the standard drug for postoperative adjuvant chemotherapy for gastric cancer in Japan [16]. However, this implies that $\mathrm{S}-1$ is also given to patients whose tumors are not sensitive to 5-FU. To address this problem, we organized a research group designated Gastric Cancer 04
(GC-04), consisting of 32 surgical institutions distributed nationwide, in the Japan Clinical Cancer Research Organization (JACCRO). GC-04 conducted this exploratory phase II trial to evaluate the clinical value of chemosensitivity testing of 5-FU in patients who received S-1 postoperatively. Our main goal was to verify whether survival is better in patients whose tumors are sensitive to 5-FU in vitro than in those insensitive to 5 -FU in vitro. The primary end point was relapse-free survival (RFS). Secondary end points included 3-year overall survival (OS) and safety. The study was performed from December 2005 to December 2013.

\section{Materials and methods}

The trial was conducted in accordance with the World Medical Association Declaration of Helsinki and Japanese Ethical Guidelines for Clinical Studies. The protocol was approved by the institutional review board of each participating hospital. Written informed consent was obtained from all patients. All members of the steering committee and the sponsor jointly designed the trial and collected the data, which were managed by the independent JACCRO GC-04 Data Center. The data were analyzed by an independent data and safety monitoring committee.

\section{Eligibility criteria}

The eligibility criteria were as follows: (1) presence of histologically proven stage II, stage IIIA, or stage IIIB gastric cancer, and stage IV gastric cancer with N3 but without hepatic, peritoneal, or distant organ metastasis; (2) treatment by $\mathrm{D} 2$ or more extensive lymph node dissection; (3) an age of 20-80 years; (4) no previous treatment for cancer; and (5) adequate organ function (a leukocyte count of at least $4,000 / \mathrm{ml}$; a platelet count of at least $100,000 / \mathrm{ml}$; a total bilirubin level of no more than $1.5 \mathrm{mg} / \mathrm{dl}$, aspartate aminotransferase and alanine aminotransferase levels of no more than 2.5 times the upper limit of the normal range; and a serum creatinine level no greater than the upper limit of the normal range). Tumor stage classification and $\mathrm{D}$ classification were in accordance with the Japanese Classification of Gastric Carcinoma (second English edition) [17]. For patients to be included in the final analysis, the in vitro sensitivity of tumor tissue to 5-FU had to be successfully evaluated by chemosensitivity testing.

\section{Drug sensitivity test}

The collagen gel droplet embedded culture drug sensitivity test (CD-DST) was used to assess in vitro sensitivity to 5-FU because it is the only commercially available method 
distributed as a kit, and various studies have demonstrated its usefulness in evaluating the in vitro chemosensitivity of fresh human tumors [18-24]. The CD-DST was performed as described previously $[25,26]$.

Briefly, a portion of each resected tumor specimen was excised and thinly sliced. Each sample was treated with dispersion enzyme cocktail EZ (Kurabo Industries, Osaka, Japan). The resulting cell suspension was transferred to collagen-coated flasks (CG-flask; Kurabo Industries) and cultured in preculture medium containing $10 \%$ fetal bovine serum at $37{ }^{\circ} \mathrm{C}$ in $5 \% \mathrm{CO}_{2}$ overnight. The collagen gel was digested with $0.05 \%$ collagenase (type I; Sigma-Aldrich Japan, Tokyo, Japan) to obtain viable cancer cells. The cancer cell suspension prepared was added to reconstructed type I collagen solution (Cellmatrix type CD; Kurabo Industries) to obtain a final cell density of $1 \times 10^{5} / \mathrm{ml}$. Three drops of the collagen-cell mixture (30 $\mu$ l per droplet) were placed in each well of a six-well plate on ice and allowed to gel at $37{ }^{\circ} \mathrm{C}$ in a $\mathrm{CO}_{2}$ incubator overnight. Subsequently, the tumor cells in the collagen gel droplet were exposed to 5-FU at concentrations corresponding to the area under the drug concentration-time curve in patients and were incubated for $120 \mathrm{~h}$. The 5-FU concentrations used were $0.2,0.4,1.0$, and $2.0 \mu \mathrm{g} / \mathrm{ml}$. After removal of the medium containing 5-FU, each well was rinsed twice with $3 \mathrm{ml}$ of Hanks balanced salt solution each time, overlaid with $4 \mathrm{ml}$ of PCM-2 medium (serum-free medium; Kurabo Industries), and incubated for 7 days. At the end of the incubation, neutral red was added to each well at a final concentration of $50 \mu \mathrm{g} / \mathrm{ml}$, and the colonies in the collagen gel droplet were fixed in $10 \%$ neutral-buffered formalin, washed with water, air-dried, and quantified by optical density image analysis. In vitro sensitivity was expressed as the $T / C$ ratio, where $T$ is the optical density of the 5-FU-treated samples on day 7 and $C$ is the optical density of nontreated controls on day 7 . The growth inhibition rate (GIR) was calculated as $1-T / C$.

In the pilot study using 31 fresh gastric cancers that were provided to the central laboratory in the initial stage of this study, we could not find any significant difference in GIR between 5-FU alone and 5-FU with CDHP, which is included in S-1, i.e., GIR of $57.5 \pm 22.5 \%$ and $64.3 \pm 17.8 \%$ for $5-\mathrm{FU}$ alone at 1.0 and $2.0 \mu \mathrm{g} / \mathrm{ml}$, respectively, versus GIR of $58.0 \pm 20.3 \%$ and $66.4 \pm 20.9 \%$ for 5 -FU at 1.0 and $2.0 \mu \mathrm{g} / \mathrm{ml}$ with CDHP, respectively. As a result, in vitro sensitivity to 5-FU was used as a surrogate of in vivo sensitivity to S-1 in this study.

\section{RNA extraction, complementary DNA synthesis, and real-time quantitative reverse transcription polymerase chain reaction}

The effect of S-1 can be modulated by the expression levels of 5-FU-related metabolic enzymes, including dihydropyrimidine dehydrogenese (DPD) [27], thymidine synthetase (TS) [28], thymidine phosphorylasa (TP), and orotate phosphoribosyltransferase (OPRT) [29].

Expression levels of TS, DPD, TP, and OPRT messenger RNA (mRNA) were measured as previously [30]. Briefly, total RNA of primary gastric cancer cells was extracted using an Isogen kit (Nippon Gene, Tokyo, Japan) according to the manufacturer's instructions. Complementary DNA was derived from each sample, and target complementary DNA sequences were amplified by quantitative polymerase chain reaction (PCR) using a fluorescence-based real-time detection method [ABI PRISM 7900 sequence detection system (TaqMan); Applied Biosystems, Foster City, CA, USA]. The PCR conditions were $50{ }^{\circ} \mathrm{C}$ for $10 \mathrm{~s}$ and $95^{\circ} \mathrm{C}$ for $10 \mathrm{~min}$, followed by 42 cycles at $95^{\circ} \mathrm{C}$ for $15 \mathrm{~s}$ and $60{ }^{\circ} \mathrm{C}$ for $1 \mathrm{~min}$. TS, DPD, TP, and OPRT mRNA levels were quantified as ratios between two measurements (gene of interest/ $\beta$-actin).

\section{Definition of the appropriate cutoff values}

Tumors with a GIR equal to the cutoff value or higher were classified as in vitro sensitive (responders), and those with lower GIRs were classified as in vitro insensitive (nonresponders). After a median follow-up time of 3 years, the hazard ratio (HR) for relapse in the responder group as compared with the nonresponder group was calculated by plotting cutoff values of the in vitro GIR from 10 to $90 \%$ with $10 \%$ increments for each of the four different in vitro 5-FU concentrations. Appropriate cutoff values were defined when the HR for relapse and the log-rank $P$ value were at their minimum.

\section{Study design and treatment}

Patients were enrolled within 6 weeks after surgery via a Web-based electronic data capture system (FLADS; Takt Systems, Tokyo, Japan) into the JACCRO GC-04 Data Center. Enrolled patients received two oral doses of $40 \mathrm{mg}$ of S-1 per square meter of body-surface area per day for 4 weeks, followed by 2 weeks of no chemotherapy (Fig. 1). During the treatment weeks, the dosages of S-1 were assigned according to body-surface area as follows: less than $1.25 \mathrm{~m}^{2}, 80 \mathrm{mg}$ daily; $1.25 \mathrm{~m}^{2}$ or greater to less than $1.5 \mathrm{~m}^{2}$, $100 \mathrm{mg}$ daily; and $1.5 \mathrm{~m}^{2}$ or greater, $120 \mathrm{mg}$ daily. This 6-week cycle treatment was repeated for 1 year after surgery. If patients had grade 3 or grade 4 hematologic toxicity or grade 2, 3, or 4 nonhematologic toxicity, the daily dose of S-1 was reduced, from 120 to $100 \mathrm{mg}$, from 100 to $80 \mathrm{mg}$, or from 80 to $50 \mathrm{mg}$, respectively.

Patients were followed up for 3 years postoperatively. Adverse events were assessed according to the Common Toxicity Criteria (version 2.0) of the National Cancer Institute. 


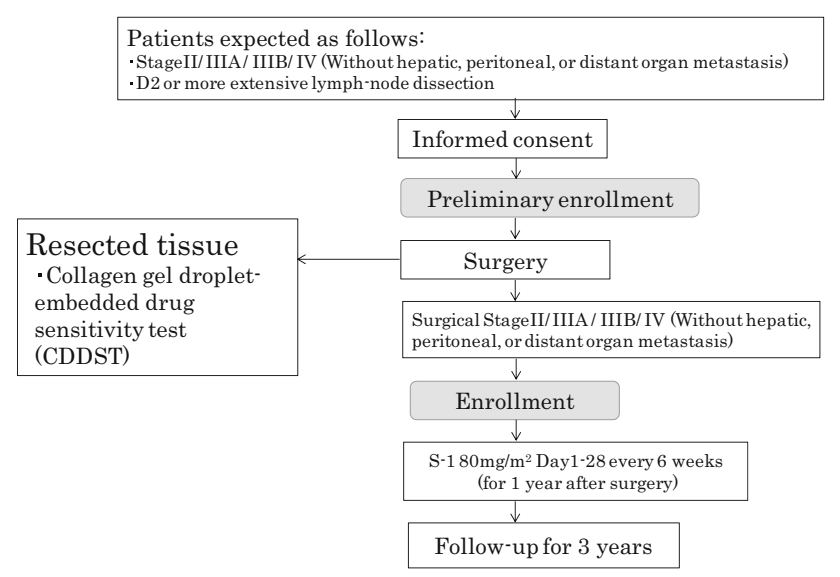

Fig. 1 Study schema

\section{Follow-up}

Patients underwent hematologic tests and clinical examinations every 2 weeks. Adverse events were evaluated every 3 months for 1 year after surgery.

The presence of recurrence was determined by means of imaging studies, including ultrasonography, computed tomography (CT), gastrointestinal radiography, and endoscopy. Patients underwent at least one type of imaging study, usually CT, at 6-month intervals during the first 2 years after surgery and then at 1-year intervals until 3 years after surgery. Peritoneal relapse was diagnosed when CT or ultrasonography identified cytology-positive ascites. Case-report forms, which included the results of follow-up tests and evaluations and the survival status of patients, were submitted to the JACCRO GC-04 Data Center $0.5,1,1.5,2$, and 3 years after surgery.

\section{Statistical analysis}

Our previous retrospective study of 128 patients with gastric cancer demonstrated that survival of the responders as determined by chemosensitivity testing, the histoculture drug-response assay [9], was significantly superior to that of the nonresponders $[31,32]$. Taking into consideration the possibilities of failure of chemosensitivity testing and inclusion of ineligible patients, we estimated that a total enrollment of 300 patients would be sufficient to reproduce similar results in the present prospective study. Because the number of days from surgery to enrollment was likely to differ among patients, we decided to calculate the OS and RFS from the date of surgery. All statistical analyses were performed using JMP 8.0 and SAS 9.2 statistical software programs (SAS, Cary, NC, USA). The 3-year RFS and OS rates were estimated using the Kaplan-Meier method, and the log-rank test was used to compare the survival curves. A Cox proportional hazards model was used to calculate the HRs. $P$ values less than 0.05 indicated statistical significance.

\section{Results}

\section{Patients and procedures}

Between December 2005 and December 2013, 311 patients were enrolled at 32 centers in Japan (Fig. 2). At enrollment, 14 patients were found to be ineligible for the following reasons: no tumor specimens available for chemosensitivity testing (ten patients), T1 cancer (two patients), enrollment before approval of the institutional review board (one patient), and laboratory test values at enrollment that did not meet the protocol requirements (one patient). In addition, 27 patients did not receive the protocol treatment of S-1. Among the tumors from the remaining 270 patients, in vitro chemosensitivity was not successfully assessed in 64 tumors for the following reasons: insufficient number of tumor cells for assay (30 patients), bacterial contamination (29 patients), low tumor cell viability (two patients), and insufficient cell growth (three patients). As a result, survival and safety were analyzed in 206 patients in whom chemosensitivity testing was successful.

\section{Characteristics of the 206 patients}

The 206 patients consisted of 151 men and 55 women with a median age of 65 years. Distribution of the disease stage, $\mathrm{T}$ stage, $\mathrm{N}$ stage, extent of lymph node dissection, ECOG performance status, type of gastrectomy, and tumor histologic type are shown in Table 1.

\section{Adverse events and treatment compliance}

Among the 206 patients who received the protocol S-1 treatment, adverse events were evaluated and classified as grade 1, 2, 3, or 4 according to the Common Toxicity Criteria (version 2.0) of the National Cancer Institute. Grade 3 or

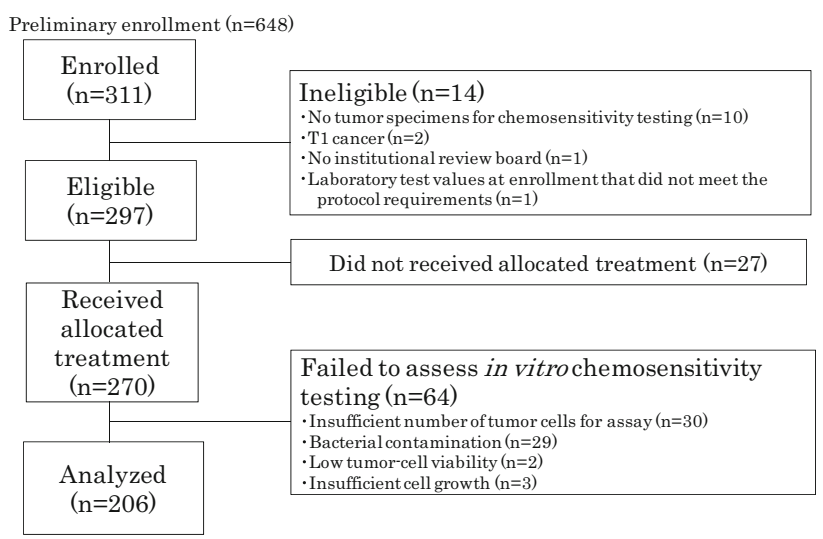

Fig. 2 CONSORT diagram 
Table 1 Patient characteristics of the 206 patients

\begin{tabular}{|c|c|}
\hline \multicolumn{2}{|l|}{ Gender } \\
\hline Male & $151(73.3 \%)$ \\
\hline Female & $55(26.7 \%)$ \\
\hline \multicolumn{2}{|l|}{ Age } \\
\hline Median & 65 years \\
\hline Range & $32-79$ years \\
\hline Mean & 63.5 years \\
\hline \multicolumn{2}{|l|}{ Body surface area $\left(\mathrm{m}^{2}\right)$} \\
\hline$<1.25$ & $4(1.9 \%)$ \\
\hline$\geq 1.25,<1.5$ & $67(32.5 \%)$ \\
\hline$\geq 1.5$ & $135(65.5 \%)$ \\
\hline \multicolumn{2}{|l|}{ Cancer stage $^{a}$} \\
\hline II & $39(18.9 \%)$ \\
\hline IIIA & $97(47.1 \%)$ \\
\hline IIIB & $58(28.2 \%)$ \\
\hline IV & $12(5.8 \%)$ \\
\hline \multicolumn{2}{|l|}{ T stage ${ }^{a}$} \\
\hline $\mathrm{T} 1$ & $2(1.0 \%)$ \\
\hline $\mathrm{T} 2$ & $52(25.2 \%)$ \\
\hline $\mathrm{T} 3$ & $135(65.5 \%)$ \\
\hline $\mathrm{T} 4$ & $17(8.3 \%)$ \\
\hline \multicolumn{2}{|l|}{$\mathrm{N}$ stage $^{\mathrm{a}}$} \\
\hline N0 & $11(5.3 \%)$ \\
\hline N1 & $109(52.9 \%)$ \\
\hline $\mathrm{N} 2$ & $79(38.3 \%)$ \\
\hline $\mathrm{N} 3$ & $7(3.4 \%)$ \\
\hline \multicolumn{2}{|l|}{ Lymph node dissection $^{\mathrm{a}}$} \\
\hline D2 & $197(95.6 \%)$ \\
\hline D3 & $9(4.4 \%)$ \\
\hline \multicolumn{2}{|l|}{ ECOG PS } \\
\hline 0 & $150(72.8 \%)$ \\
\hline 1 & $56(27.2 \%)$ \\
\hline \multicolumn{2}{|l|}{ Type of gastrectomy } \\
\hline Distal & $124(60.2 \%)$ \\
\hline Total & $82(39.8 \%)$ \\
\hline \multicolumn{2}{|l|}{ Tumor histology } \\
\hline Intestinal type & $81(39.3 \%)$ \\
\hline Diffuse type & $123(59.7 \%)$ \\
\hline Neuroendocrine cell carcinoma & $1(0.5 \%)$ \\
\hline Unknown & $1(0.5 \%)$ \\
\hline
\end{tabular}

ECOG PS Eastern Cooperative Oncology Group performance status

a Japanese Classification of Gastric Cancer 13th edition

grade 4 adverse events included neutropenia (10.7\%), diarrhea $(5.9 \%)$, mechanical ileus $(4.1 \%)$, leukopenia $(2.6 \%)$, anorexia $(2.6 \%)$, anemia $(2.2 \%)$, skin rash $(2.2 \%)$, and stomatitis (2.2\%). S-1 treatment was continued for at least 3 months in 183 patients $(88.8 \%)$, for at least 6 months in 154 patients $(74.8 \%)$, for at least 9 months in 139 patients $(67.0 \%)$, and for 12 months in 99 patients
$(48.1 \%)$. The dose of S-1 was decreased in $76(36.9 \%)$ of the 206 patients who received the protocol treatment. Of the 99 patients who received the treatment for 12 months, the dose was decreased in 41 patients $(41.4 \%)$.

\section{OS and RFS}

On the basis of follow-up data updated as of December 31, 2013, the median follow-up from the time of surgery was 3.2 years in the 206 patients. Forty-seven patients had died. The causes of death were relapse in 39 patients, other cancer in two patients, causes other than cancer in four patients, and unknown causes in two patients. Recurrent diseases occurred in 51 patients. The OS and RFS rates in the 206 patients were $96.1 \%$ and $86.8 \%$, respectively, at 1 year, $87.7 \%$ and $76.9 \%$, respectively, at 2 years, and $80.6 \%$ and $71.9 \%$, respectively, at 3 years.

\section{Messenger RNA levels of TS, DPD, TP, and OPRT}

Gene expression levels of TS, DPD, TP, and OPRT were successfully measured in these 206 tumors. However, mRNA levels of none of these biomarkers correlated with the GIR induced by 5-FU in each of four different 5-FU concentrations (data not shown).

\section{Association between in vitro sensitivities to 5-FU and survival of patients who received S-1 treatment}

One of the main purposes of this study was to investigate appropriate cutoff values for classifying patients as likely responders or nonresponders. As described in "Materials and methods," four different in vitro 5-FU concentrations, which were comparable to clinically achievable plasma 5-FU concentrations, were used to assess the in vitro sensitivity of tumor cells to 5-FU in the CD-DST. The correlation between in vitro chemosensitivity and survival outcome after a 3-year follow-up period is summarized with the forest plot in Fig. 3 in relation to the HR, 3-year RFS, and log-rank $P$ value between the responder and nonresponder groups. As shown in Fig. 3, an HR of less than 0.4 with narrow $95 \%$ confidence intervals (CIs) and significant $P$ values strongly suggested that appropriate cutoff values for dividing patients into responders and nonresponders were in vitro GIRs of 20-30 \% at an in vitro 5-FU concentration of $0.2 \mu \mathrm{g} / \mathrm{ml}, 30-40 \%$ at $0.4 \mu \mathrm{g} / \mathrm{ml}$, $50-60 \%$ at $1.0 \mu \mathrm{g} / \mathrm{ml}$, and $60-70 \%$ at $2.0 \mu \mathrm{g} / \mathrm{ml}$. These results indicated that the appropriate cutoff values are substantially influenced by the in vitro drug concentration and can be defined in some, albeit not narrow, ranges.

When these cutoff values were applied, as shown in Fig. 3, responders had significantly better survival than nonresponders for each of the four different in vitro 5-FU 
Fig. 3 Forest plot to identify appropriate cutoff values for each of the four in vitro 5-fluorouracil concentrations for a total of 206 patients. $C I$ ratio, Non nonresponders, Res responders, $R F S$ relapse-free survival confidence interval, $H R$ hazard

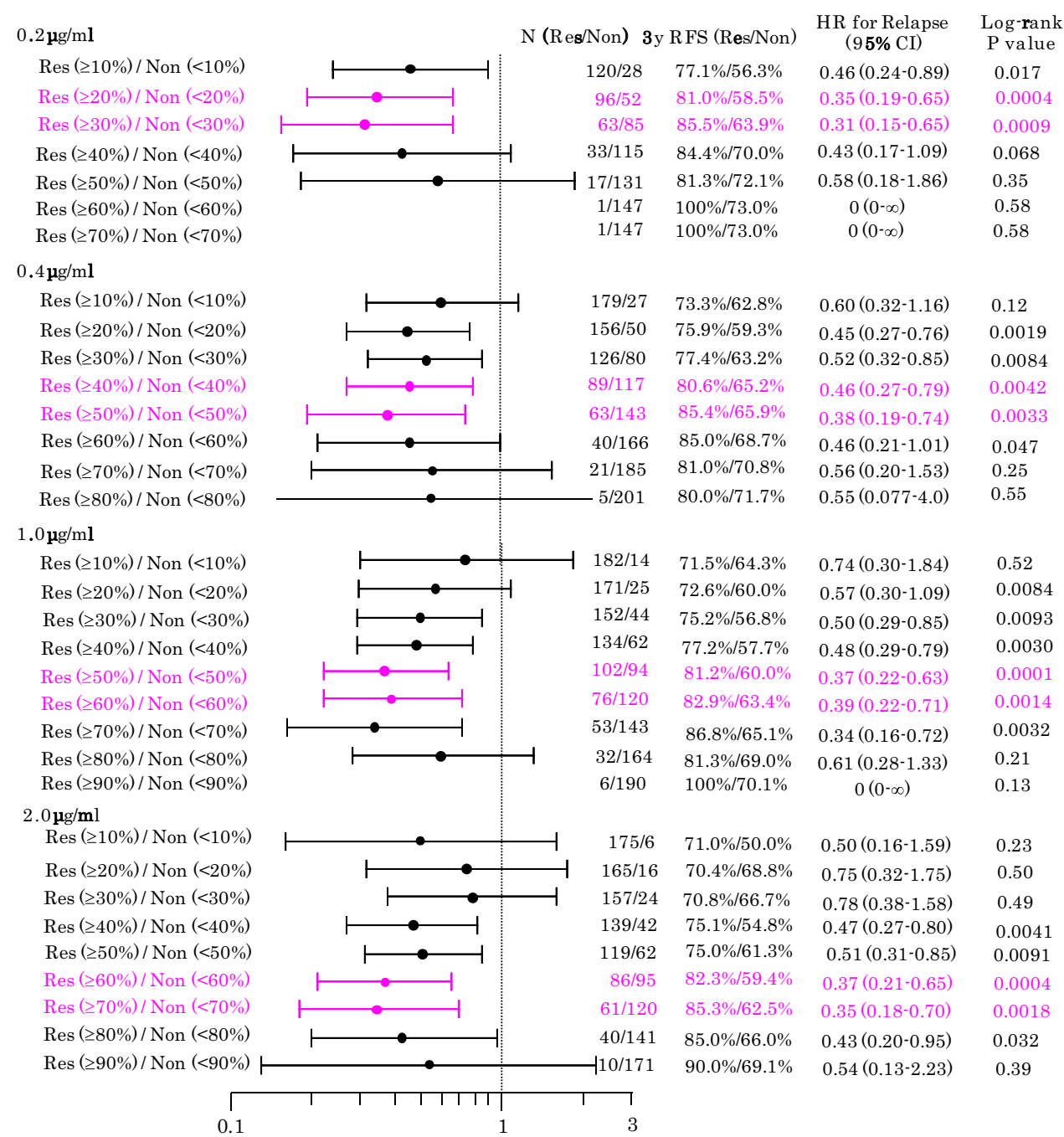

concentrations, whereas no significant differences were observed in background clinical characteristics, except for age, between the responder and nonresponder groups. As an example, when an in vitro GIR of $60 \%$ at an in vitro 5 -FU concentration of $1.0 \mu \mathrm{g} / \mathrm{ml}$ was applied as a cutoff value, the HR for tumor relapse in the 76 responders, compared with the 120 nonresponders, was 0.39 (95\% CI $0.22-0.71 ; P=0.0014)$. The 3 -year RFS rate was $82.9 \%$ (95\% CI 74.4-91.3\%) in the responder group and $63.4 \%$ (95\% CI 54.7-72.1\%) in the nonresponder group (Fig. 4), whereas there were no significant differences in background clinical characteristics, except for age, between the responder and nonresponder groups as in Table 2. In addition, as indicated in Table 2, there was no significant difference in relapse sites between the two groups.

The HR for tumor relapse of responders compared with nonresponders was 0.24 (95\% CI $0.08-0.68)$ in 113 patients with $\mathrm{N} 0$ or $\mathrm{N} 1$ lymph node metastasis and 0.58

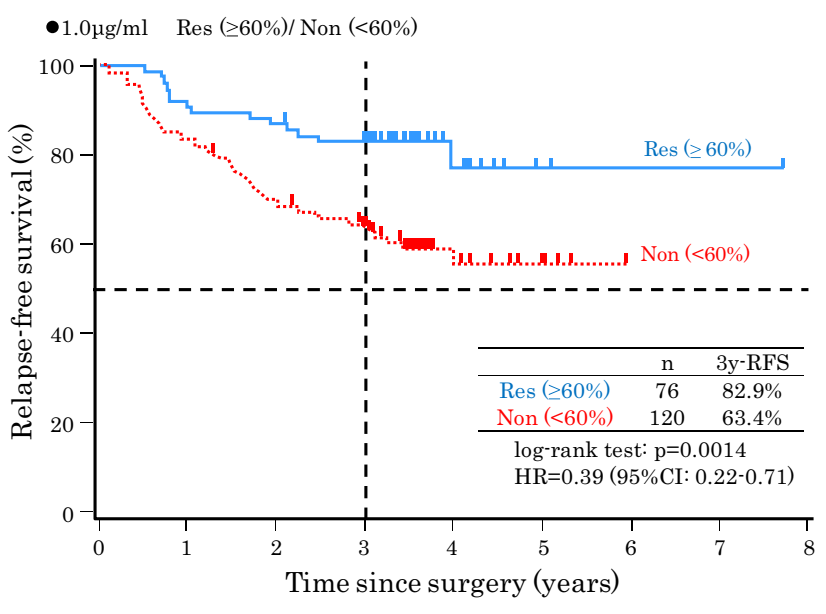

Fig. 4 Relapse-free survival (RFS) of responder (Res) and nonresponder (Non) groups classified by a growth inhibition rate of $60 \%$ at an in vitro 5-fluorouracil concentration of $1.0 \mu \mathrm{g} / \mathrm{ml}$. CI confidence interval, $H R$ hazard ratio 
Table 2 Comparison of background clinical characteristics of responders and nonresponders classified by a growth inhibition rate of $60 \%$ at a 5 -fluorouracil concentration of $1.0 \mu \mathrm{g} / \mathrm{ml}$

\begin{tabular}{|c|c|c|c|}
\hline Characteristic & Responders $(n=76)$ & Non responders $(n=120)$ & $P$ \\
\hline \multicolumn{3}{|l|}{ Gender } & \multirow[t]{3}{*}{0.09} \\
\hline Male & $61(80.3 \%)$ & $83(69.2 \%)$ & \\
\hline Female & $15(19.7 \%)$ & $37(30.8 \%)$ & \\
\hline Age & 62.5 years (32-78 years) & 67.0 years (33-79 years) & 0.01 \\
\hline \multicolumn{3}{|l|}{ Cancer stage $^{\mathrm{a}}$} & \multirow[t]{4}{*}{0.54} \\
\hline II & $14(18.4 \%)$ & $23(19.2 \%)$ & \\
\hline III & $56(73.7 \%)$ & $92(76.7 \%)$ & \\
\hline IV & $6(7.9 \%)$ & $5(4.2 \%)$ & \\
\hline \multicolumn{3}{|l|}{ Tumor stage $^{\mathrm{a}}$} & \multirow[t]{5}{*}{0.42} \\
\hline $\mathrm{T} 1$ & $0(0 \%)$ & $2(1.7 \%)$ & \\
\hline $\mathrm{T} 2$ & $22(28.9 \%)$ & $27(22.5 \%)$ & \\
\hline $\mathrm{T} 3$ & $47(61.8 \%)$ & $83(69.2 \%)$ & \\
\hline $\mathrm{T} 4$ & $7(9.2 \%)$ & $8(6.7 \%)$ & \\
\hline \multicolumn{3}{|l|}{$\mathrm{N}_{\text {stage }}{ }^{\mathrm{a}}$} & \multirow[t]{5}{*}{0.95} \\
\hline No & $4(5.3 \%)$ & $7(5.8 \%)$ & \\
\hline N1 & $38(50.0 \%)$ & $64(53.3 \%)$ & \\
\hline $\mathrm{N} 2$ & $31(40.8 \%)$ & $45(37.5 \%)$ & \\
\hline N3 & $3(3.9 \%)$ & $4(3.3 \%)$ & \\
\hline \multicolumn{3}{|c|}{ Type of lymph node dissection ${ }^{a}$} & \multirow[t]{3}{*}{0.29} \\
\hline D2 & $71(93.4 \%)$ & $116(96.7 \%)$ & \\
\hline D3 & $5(6.6 \%)$ & $4(3.3 \%)$ & \\
\hline \multicolumn{3}{|l|}{ ECOG PS } & \multirow[t]{3}{*}{0.18} \\
\hline 0 & $51(67.1 \%)$ & $91(75.8 \%)$ & \\
\hline 1 & $25(32.9 \%)$ & $29(24.2 \%)$ & \\
\hline \multirow[t]{2}{*}{$\mathrm{RDI}^{\mathrm{b}}$} & $70.2 \%$ & $64.3 \%$ & \multirow[t]{2}{*}{0.29} \\
\hline & $(0.9-186 \%)$ & $(0.4-119 \%)$ & \\
\hline \multicolumn{3}{|c|}{ Type of gastrectomy } & \multirow[t]{3}{*}{0.80} \\
\hline Total & $47(61.8 \%)$ & $72(60.0 \%)$ & \\
\hline Distal & $29(38.2 \%)$ & $48(40.0 \%)$ & \\
\hline \multicolumn{3}{|l|}{ Tumor histology } & \multirow[t]{5}{*}{0.54} \\
\hline Intestinal type & $27(35.5 \%)$ & $52(43.3 \%)$ & \\
\hline Diffuse type & $48(63.2 \%)$ & $67(55.8 \%)$ & \\
\hline Unknown & $1(1.3 \%)$ & $1(0.8 \%)$ & \\
\hline Sites of relapse & $n=12$ & $n=43$ & \\
\hline Local & $0(0 \%)$ & $5(11.6 \%)$ & 0.22 \\
\hline Peritoneum & $4(33.3 \%)$ & $19(44.2 \%)$ & 0.50 \\
\hline Liver & $4(33.3 \%)$ & $11(25.6 \%)$ & 0.59 \\
\hline Distant & $4(33.3 \%)$ & $7(16.3 \%)$ & 0.19 \\
\hline Lymph node & $4(33.3 \%)$ & $6(14.0 \%)$ & 0.12 \\
\hline
\end{tabular}

There were no significant differences in the background clinical characteristics, except for age, between the responder and nonresponder groups, even when classified by any other defined cutoff values (data not shown)

ECOG PS Eastern Cooperative Oncology Group performance status, RDI relative dose intensity

a Japanese Classification of Gastric Cancer 13th edition

b $\mathrm{RDI}=$ actual intake of doses/total protocol doses of S-1 for 1 year (\%)

c Some patients had plural relapses 
(95\% CI $0.25-1.23)$ in 83 patients with N2 or N3 lymph node metastasis. It was 0.18 (95\% CI 0.00-1.01) in 47 patients with stage II disease and 0.38 (95 \% CI 0.18-0.74) in 148 patients with stage III disease.

\section{Discussion}

The CD-DST is a chemosensitivity test wherein isolated tumor cells are embedded in collagen droplets. This threedimensional culture system has the following advantages over other conventional methods such as 3-(4,5dimethylthiazol-2-yl)-2,5-diphenyltetrazolium bromide [33] and ATP [34] assays: the ability to use small specimens, the ability to assess the effect of anticancer drugs at physiological concentrations, and the ability to eliminate the masking effect caused by fibroblast contamination in culture with the aid of an image analysis system [26, 35].

The efficacy of the CD-DST in cancer treatment has previously been demonstrated in various malignant human tumors, including gastric cancer $[18,19]$ and other malignancies [20-24]. However, as recent controversial discussion on chemosensitivity testing for human tumor specimens has indicated [36-42], the studies with the CDDST also had significant limitations, including small sample sizes, the lack of prospective studies, and the lack of clear cutoff values to distinguish chemotherapy sensitivity from resistance. Accordingly, we conducted this exploratory phase II trial in a multicenter setting to evaluate the clinical value of chemosensitivity testing for 5-FU in patients who received $\mathrm{S}-1$ postoperatively. Our main goal was to verify whether survival is better in patients whose tumors are sensitive to 5-FU in vitro than in those with tumors insensitive to 5-FU in vitro, when appropriate cutoff values to classify the patients as responders and nonresponders were defined.

As one of the major findings of the present study, in vitro chemosensitivity testing of gastric cancer samples using the CD-DST proved to be a feasible method and yielded a success rate of $76 \%$ (206 of 270 samples). Major reasons for unsuccessful assessment of the remaining 64 samples included insufficient number of tumor cells for assay (30 samples) and bacterial contamination (29 samples), as shown in Fig. 2. Both problems may possibly be attributed to the limitations arising from a multicenter setting, such as the inconsistent manner of the handling of tumor samples or the time to transport samples to the laboratory. As a result, there still remains some room for improvement of these technical issues. Also, the test results were obtained within 7 days in all cases, suggesting that the CD-DST may be a useful method in prospective studies to evaluate the clinical significance of sensitivity-test-guided chemotherapy in an adjuvant setting.
As one of accompanying studies in this trial, mRNA expression levels of TS, DPD, TP, and OPRT were quantified by reverse transcription PCR by use of prepared fresh tumor cells. No correlation was found between the mRNA expression of those enzymes and in vitro 5-FU sensitivity, suggesting that it is not possible to predict 5-FU sensitivity solely on the basis of gene expression of the enzymes considered in this study.

Our second finding of this trial was that appropriate cutoff values classifying patients as responders or nonresponders were able to be defined by the calculated HR for tumor relapse and log-rank $P$ values. The 3 -year RFS rate was significantly better in the responder group than in the nonresponder group when the defined appropriate cutoff values for each in vitro 5-FU concentration were applied. The cutoff values of 50-60\% at a 5-FU concentration of $1.0 \mu \mathrm{g} / \mathrm{ml}$ were already used in previously published reports $[18,19,21]$, in which those values were retrospectively determined. Our results verify the finding of the previous studies that there is a direct association between in vitro sensitivity and therapy outcome.

The primary end point of this study was 3-year RFS, the same as in the CLASSIC trial, which was an adjuvant chemotherapy study recently conducted in South Korea [43]. The CLASSIC trial successfully demonstrated a significant survival benefit from adjuvant capecitabine and oxaliplatin chemotherapy compared with surgery alone in patients with stage II-IIIB gastric cancer after D2 surgery. Additionally, in ACTS-GC, whose primary end point was 5-year OS, the 3-year RFS rates were 72.4 and $61.1 \%$ and the 5-year OS rates were 71.7 and $61.1 \%$ in the S-1 group and the surgery-only group, respectively. These findings, in addition to the results of this study, may justify the currently controversial use of the 3-year RFS as the primary end point in clinical trials of adjuvant chemotherapy for potentially curable gastric cancer.

Since the definition of RFS is crucial and very delicate in this set of patients, the follow-up method used was the same as that in ACTS-GC. The absolute number of patients whose relapse was firstly identified was 23 during the first 1 year, 20 between 1 and 2 years, and 8 between 2 and 3 years after surgery in this study, meaning that most of the recurrence occurred within 2 years after surgery. This seems to justify the follow-up method used in the current study.

There were no significant differences between the responder and nonresponder groups in the background clinical characteristics, except for age. The responder group had younger patients than the nonresponder group. However, as also demonstrated in Table 2, the relative dose intensity was almost comparable between these two groups. As a result, the better survival in responders did not seem to be explained by $\mathrm{S}-1$ treatment compliance. 
The subset analysis of the HR for tumor relapse of responders compared with nonresponders with respect to tumor stages and lymph node metastases suggested a tendency for a more favorable effect of S1 treatment on patients with an earlier stage of tumor development and of extent of lymph node metastasis, as indicated by ACTS-GC. However, this was not definitely confirmed in this study, probably because of insufficient number of enrolled patients.

In conclusion, to the best of our knowledge, the present phase II study conducted in a multicenter setting is the first large clinical trial to evaluate prospectively the clinical significance of chemosensitivity testing in patients with gastric cancer. Use of the CD-DST may contribute to the proper selection of candidates for chemotherapy and may aid in the reduction of unnecessary adverse events in patients insensitive to 5-FU. This encouraging finding needs further evaluation in a randomized controlled phase III trial to prove that sensitivity-test-guided chemotherapy may provide greater survival benefit than conventional empirical chemotherapy in patients with locally advanced gastric cancer in an adjuvant setting.

Acknowledgments The present study was started by an initial principal investigator, Tetsuro Kubota, late professor of Keio University School of Medicine, Tokyo. Since he passed away halfway through this study in January 2009, all participating members have agreed to call this study the "Kubota memorial trial" in appreciation of his great contribution to the research field of anticancer drug sensitivity.

Conflict of interest The following authors have a financial or other interest that is relevant to the subject matter considered in this article:Consultant or advisory role: Masashi Fujii, Taiho Pharmaceutical; Toshifusa Nakajima, Taiho Pharmaceutical; Yuh Sakata, Taiho Pharmaceutical, Otsuka Holdings. Stock ownership: Masashi Fujii, Otsuka Holdings. Honorarium: Nobuhiko Tanigawa, Taiho Pharmaceutical; Hiroki Yamaue, Taiho Pharmaceutical; Shinichi Sakuramoto, Taiho Pharmaceutical; Takao Inada, Taiho Pharmaceutical; Yasuhiro Kodera, Taiho Pharmaceutical; Yuko Kitagawa, Taiho Pharmaceutical; Yuh Sakata, Taiho Pharmaceutical; Atsuhi Nashimoto, Taiho Pharmaceutical; Toshiharu Yamaguchi, Taiho Pharmaceutical; Toshifusa Nakajima, Taiho Pharmaceutical. Research funding: Hiroki Yamaue, Taiho Pharmaceutical; Yasuhiro Kodera, Taiho Pharmaceutical; Yuko Kitagawa, Taiho Pharmaceutical; Yuh Sakata, Taiho Pharmaceutical.

Open Access This article is distributed under the terms of the Creative Commons Attribution 4.0 International License (http://creativecommons.org/licenses/by/4.0/), which permits unrestricted use, distribution, and reproduction in any medium, provided you give appropriate credit to the original author(s) and the source, provide a link to the Creative Commons license, and indicate if changes were made.

\section{Appendix}

Members of the JACCRO GC04 group were as follows: Steering Committee: N. Tanigawa, H. Yamaue, S. Sakuramoto, Y. Kodera, Y. Kitagawa, K. Omura, M. Terashima,
Y. Sakata, K. Chin, A. Nashimoto, T. Yamaguchi, E. Nomura, M. Takeuchi, M. Fujii, T. Nakajima. Independent Data and Safety Monitoring Committee: Y. Shimada, M. Takeuchi, T. Sano. Medical adviser: T. Nakajima. Statistical analysis: M. Takeuchi. Principal investigators (all in Japan): G. Wakabayashi (Iwate Medical University Hospital), M. Gotoh (Fukushima Medical University Hospital), A. Nashimoto (Niigata Cancer Center Hospital), H. Kuwano (Gunma University Hospital), H. Kato (Dokkyo Medical University Hospital), T. Inada (Tochigi Cancer Center), Y. Yasuda (Jichi Medical University Hospital), S. Sakuramoto (Saitama Medical University International Medical Center), T. Ochiai (Tobu Chiiki Hospital), T. Yamaguchi (Cancer Institute Hospital of JFCR), Y. Kitagawa (Keio University Hospital), Y. Isobe (Tokyo Medical Center), A. Tokunaga (Nippon Medical School Musashi Kosugi Hospital), M. Terashima (Shizuoka Canter Center), T. Yoshikawa (Kanagawa Cancer Center), S. Ito (Aichi Cancer Center Hospital), T. Hara (Kouseiren Takaoka Hospital), K. Yokoi (Kanazawa University Hospital), N. Tanigawa (Osaka Medical College), S. Morita (Hirakata City Hospital), A. Hara (Saiseikai Suita Hospital), K. Hirakawa (Osaka City University Hospital), K. Iwase (Osaka General Medical Center), I. Miyashiro (Osaka Medical Center for Cancer and Cardiovascular Diseases), E. Nakata (Otori Stomachi and Intestine Hospital), T. Nitta (Shiroyama Hospital), J. Matsuyama (Yao Municipal Hospital), H. Yamaue (Wakayama Medical University), K. Yoshikawa (Tokushima University Hospital), Y. Maehara (Kyushu University Hospital), Y. Kitajima (Saga University Hospital), S. Natsugoe (Kagoshima University Hospital).

\section{References}

1. Sakuramoto S, Sasako M, Yamaguchi T, Kinoshita T, Fujii M, Nashimoto A, et al. Adjuvant chemotherapy for gastric cancer with S-1, on oral fluoropyrimidine. $N$ Engl $J$ Med. 2007;357:1810-20.

2. Sasako M, Sakuramoto S, Takai H, Kinoshita T, Furukawa H, Yamaguchi T, et al. Five-year outcomes of a randomized phase III trial comparing adjuvant chemotherapy with $S-1$ versus surgery alone in stage II or III gastric cancer. J Clin Oncol. 2011;29:4387-93.

3. Tanigawa N, Mizuno Y, Hashimura T, Honda K, Satomura K, Hikasa Y, et al. Comparison of drug sensitivity among tumor cells within a tumor, between primary and metastases, and between different metastases in the human tumor-colony forming assay. Cancer Res. 1984;44:2309-12.

4. Trope C, Hakansson L, Dencker H. Heterogeneity of human adenocarcinomas of the colon and the stomach as regards sensitivity to cytostatic drugs. Neoplasma. 1975;22:423-30.

5. Blorklund A, Hakansson L, Stenstam B, Trope C, Akerman M. On heterogeneity of non-Hodgkin's lymphomas as regards sensitivity to cytostatic drugs. An in vitro study. Eur J Cancer. $1980 ; 16: 647-54$. 
6. Tsuruo T, Fidler IJ. Differences in drug sensitivity among tumor cells from parental tumors, selected varients, and spontaneous metastases. Cancer Res. 1981;41:3058-64.

7. Tanigawa N, Kern DH, Hikasa Y, Morton DL. Rapid assay for evaluating the chemosensitivity of human tumors in soft agar culture. Cancer Res. 1982;42:2159-64.

8. Sondak VK, Bertelsen CA, Tanigawa N, Hilderbrand-Zanki SU, Morton DL, Kern DH. Clinical correlation with chemosensitivities measured in a rapid thymidine incorporation assay. Cancer Res. 1984;44:1725-32.

9. Vescio RA, Connors KM, Kubota T, Hoffman RM. Correlation of histology and drug response of human tumors grown in nativestate three-dimensional histoculture and in nude mice. Proc Natl Acad Sci U S A. 1991;88:5163-6.

10. Yamaue H, Tanimura H, Noguchi K, Hotta T, Tani M, Tsunoda $\mathrm{T}$, et al. Chemosensitivity testing of fresh human gastric cancer with highly purified tumour cells using the MTT assay. Br J Cancer. 1992;66:794-9.

11. Weisenthal LM. Clinical correlations for cell culture assays based on the concept of total cell kill. Contrib Gynecol Obstet. 1994;19:82-90.

12. Yamaue H, Tanimura H, Nakamori M, Noguchi K, Iwahashi M, Tani M, et al. Clinical evaluation of chemosensitivity testing for patients with colorectal cancer using MTT assay. Dis Colon Rectum. 1996;39:416-22.

13. Kondo T, Yamauchi M, Tominaga S. Evaluation of usefulness of in vitro drug sensitivity testing for adjuvant chemotherapy of stomach cancer. Int J Clin Oncol. 2000;5:174-82.

14. Cortazar P, Johnson BE. Reviews of the efficacy of individualized chemotherapy selected by in vitro drug sensitivity testing for patients with cancer. J Clin Oncol. 1999;17:1625-31.

15. Samson DJ, Seidenfeld J, Ziegler K, Aronson N. Chemotherapy sensitivity and resistance assays: a systemic review. J Clin Oncol. 2004;22:3618-30.

16. Japanese Gastric Cancer Association: Gastric cancer treatment guidelines 2010. Tokyo: Kanehara; 2010.

17. Association Japanese Gastric Cancer. Japanese classification of gastric carcinoma -2nd English edition -. Gastric Cancer. 1998;1:10-24.

18. Maejima K, Tokunaga A, Kiyama T, Kanno H, Bou H, Watanabe $\mathrm{M}$, et al. Chemosensitivity test for 5-fluorouracil and 5-chloro2,4-dihydroxypyridine predicts outcome of gastric cancer patients receiving S-1 postoperatively. Gastric Cancer. 2010;13:231-7.

19. Naitoh H, Yamamoto H, Murata S, Kobayashi H, Inoue K, Tani T. Stratified phase II trial to establish the usefulness of the collagen gel droplet embedded culture-drug sensitivity test (CDDST) for advanced gastric cancer. Gastric Cancer. 2014;7:630-7.

20. Gu F, Ma Y, Zhang Z, Zhao J, Kobayashi H, Zhang L, et al. Expression of Stat 3 and Notch1 is associated with cisplatin resistance in head and neck squamous cell carcinoma. Oncol Rep. 2010;23:671-6.

21. Higashiyama M, Oda K, Okami J, Maeda J, Kodama K, Takami $\mathrm{K}$, et al. In vitro chemosensitivity test using the collagen gel droplet-embedded drug sensitivity test (CD-DST) for malignant pleural mesothelioma: possibility of clinical application. Ann Thorac Cardiovasc Surg. 2008;14:355-62.

22. Higashiyama M, Oda K, Okami J, Maeda J, Kodama K, Imamura F, et al. Predication of chemosetherapeutic effect on postoperative small cell lung cancer patients. Lung Cancer. 2010;68:472-7.

23. Kawamura M, Gika M, Abiko T, Inoue Y, Oyama T, Izumi Y, et al. Clinical evaluation of chemosensitivity test for patients with unresectable non-small cell lung cancer (NSCLC) using collagen gel droplet embedded culture-drug sensitivity test (CD-DST). Cancer Chemother Pharmacol. 2007;59:507-13.
24. Takamura Y, Kobayashi $\mathrm{H}$, Taguchi T, Motoyama K, Inaji H, Noguchi S. Prediction of chemotherapeutic response by collagen gel droplet embedded culture drug sensitivity test in human breast cancers. Int J Cancer. 2002;98:450-5.

25. Kobayashi H, Higashiyama M, Minamigawa K, Tanisaka K, Takano $\mathrm{T}$, Yokouchi $\mathrm{H}$, et al. Examination of in vitro chemosensitivity test using collagen gel droplet culture method with colorimetric endpoint quantification. Jpn J Cancer Res. 2001;92:203-10.

26. Kobayashi H. Development of a new in vitro chemosensitivity test using collagen gel droplet embedded culture and image analysis for clinical usefulness. Recent Results Cancer Res. 2003;161:48-61.

27. Heggie GD, Sommadossi JP, Cross DS, Huster WJ, Diasio RB. Clinical pharmacokineticsof 5-fluorouracil and its metabolites in plasma, urine, and bile. Cancer Res. 1987;47:2203-6.

28. Danenberg PV. Thymidilate synthase - a target enzyme in cancer chemotherapy. Biochem Biophys Acta. 1977;473:73-92.

29. Peters GJ, Laurensse E, Leyva A, Lankelma J, Pnedo HM. Sensivity of human, murine, and rat cells drug metabolizing enzymes. Cancer Res. 1986;46:20-8.

30. Kodera Y, Ito S, Fujiwara M, Mochizuki Y, Nakayama G, Ohashi $\mathrm{N}$, et al. Gene expression of 5-fluorouracil metabolic enzymes in primary gastric cancer: correlation with drug sensitivity against 5-fluorouracil. Cancer Lett. 2007;252:307-13.

31. Kubota T, Sasano N, Abe O, Nakao I, Kawamura E, Saito T, et al. Potential of the histoculture drug-response assay to contribute to cancer patient survival. Clin Cancer Res. 1995;1:1537-43.

32. Furukawa T, Kubota T, Hoffman RM. Clinical applications of the histoculture drug response assay. Clin Cancer Res. 1995;1:305-11.

33. Xu JM, Song ST, Tang ZM, Jiang ZF, Liu XQ, Zhou L, et al. Predictive chemotherapy of advanced breast cancer directed by MTT assay in vitro. Breast Cancer Res Treat. 1999;53:77-85.

34. Kurbacher CM, Cree IA, Bruckner HW, Brenne U, Kurbacher JA, Muller K, et al. Use of an ex vivo ATP luminescence assay to direct chemotherapy for recurrent ovarian cancer. Anticancer Drugs. 1998;9:51-7.

35. Kobayashi H, Tanisaka K, Doi O, Kodama K, Higashiyama M, Nakagawa $\mathrm{H}$, et al. An in vitro chemosensitivity test for solid human tumors using collagen gel droplet embedded cultures. Int J Oncol. 1997; 11:449-55.

36. Schrag D, Garewal HS, Burstein HJ, Samson DJ, Von Hoff DD, Somerfield. ASCO working group on chemotherapy sensitivity and resistance assays. American Society of Clinical Oncology technology assessment: chemotherapy sensitivity and resistance assays. J Clin Oncol. 2004;12:3631-8.

37. Fruehauf JP, Alberts DS. In vitro drug resistance versus chemosensitivity: two sides of different coins. J Clin Oncol. 2005;23:3646-8.

38. Ugurel S, Schadendorf D, Pfohler C, Neuber K, Thoelke A, Ulrich $\mathrm{J}$, et al. In vitro drug sensitivity predicts response and survival after individualized sensitivity-directed chemotherapy in metastatic melanoma: a multicenter phase II trial of the Dermotologic Cooperative Oncology Group. Clin Cancer Res. 2006;12:5454-63.

39. Kodera Y, Ito S, Fujiwara M, Mochizuki Y, Ohashi N, Ito Y, et al. In vitro chemosensitivity test to predict chemosensitivity for paclitaxel, using human gastric carcinoma tissues. In J Clin Oncol. 2006;11:449-53.

40. Mi Z, Holmes FA, Hellerstedt B, Pippen J, Collea R, Backner A, et al. Feasibility assessment of a chemoresponse assay to predict pathologic response in neoadjuvant chemotherapy for breast cancer patients. Anticancer Res. 2008;28:1733-40. 
41. Wu B, Zhu JS, Zhang Y, Shen WM, Zhang Q. Predictive value of MTT assay as an in vitro chemosensitivity testing for gastric cancer: one institution's experience. World J Gastroenterol. 2008;14:3064-8.

42. Burstein HJ, Mangu PB, Somerfield MR, Schrag D, Samson D, Holt L, et al. American Society of Clinical Oncology clinical practice guideline update on the use of chemotherapy sensitivity and resistance assays. J Clin Oncol. 2011;29:3328-30.

43. Bang YJ, Kim YW, Yang HK, Chung HC, Park YK, Lee KH, Lee $\mathrm{KW}$, Kim YH, Noh SH. Adjuvant capecitabine and oxaliplatin for gastric cancer after D2 gastrectomy (CLASSIC): a phase 3 openlabel, randomised controlled trial. Lancet. 2012;379:315-21. 